\title{
Nonalcoholic fatty liver disease and type 2 diabetes: a burgeoning problem with unclear solutions
}

\author{
Mariana Lazo ${ }^{1}$, Jeanne M. Clark ${ }^{1,2}$ \\ ${ }^{1}$ Division of General Internal Medicine, School of Medicine, Johns Hopkins University, Baltimore, MD, USA; ${ }^{2}$ Division of Gastroenterology \& \\ Hepatology, Johns Hopkins University School of Medicine, Baltimore, MD, USA \\ Correspondence to: Jeanne M. Clark. 2024 E. Monument St. Suite 2-600, Baltimore, MD 21205, USA. Email: jclark1@jhmi.edu. \\ Provenance and Peer Review: This article was commissioned by the editorial office, Hepatobiliary Surgery and Nutrition. The article did not undergo \\ external peer review. \\ Comment on: Younossi ZM, Golabi P, de Avila L, et al. The global epidemiology of NAFLD and NASH in patients with type 2 diabetes: A systematic \\ review and meta-analysis. J Hepatol 2019;71:793-801.
}

Submitted Nov 10, 2019. Accepted for publication Nov 30, 2019.

doi: 10.21037/hbsn.2019.11.28

View this article at: http://dx.doi.org/10.21037/hbsn.2019.11.28

The association between chronic liver disease and diabetes has been known for long time, with reports in the literature as early as in 1952 (1). Currently, a bidirectional association between type 2 diabetes and nonalcoholic fatty liver disease (NAFLD) has become more widely accepted, probably as a result of the common risk factors (e.g., obesity) and pathophysiology (e.g., insulin resistance) (2).

Compared to the very early reports in which most of the evidence was presented as case reports $(3,4)$, the current context has vastly changed. With a long and sustained epidemic of obesity and type 2 diabetes in the US and worldwide, studies documenting the association between diabetes and NAFLD have been increasing in number and size. Given the accumulation of published reports, a key step is to summarize the literature in order to obtain more precise estimates of disease prevalence and to understand gaps in the literature. Indeed, a recent systematic review and meta-analysis documented a high burden in the general population, with $25.2 \%$ of the adult population worldwide having NAFLD (5). As a follow up study, the same group conducted a similar meta-analysis, published in 7 Hepatology in June 2019, focused on establishing the burden of NAFLD among people with type 2 diabetes (6).

In this study, the authors gathered information from reports worldwide (80 studies, involving 49,419 participants) from 1989 to 2018. The findings are, as suspected, worrisome. Worldwide, more than half $(55.6 \%)$ of adults with type 2 diabetes are estimated to have hepatic steatosis (the earliest manifestation of NAFLD). In addition, more than one third $(37.3 \%)$ have nonalcoholic steatohepatitis (NASH) and $17 \%$ have fibrosis. Importantly, estimates of NASH and fibrosis were derived from 10 studies conducted between 2004 and 2018 and included 892 adults with type 2 diabetes and NAFLD who had a liver biopsy. Participants with NAFLD had a mean age of 59 years old, $53 \%$ were male and the mean body mass index (BMI) was $28 \mathrm{~kg} / \mathrm{m}^{2}$.

The study also highlights substantial differences in the prevalence of hepatic steatosis among people with type 2 diabetes across regions of the world: with the lowest prevalence in Africa (32\%) and highest in Europe (72\%). However, based on results presented in the supplemental material, and from what we know with respect to the genetic underpinning of NAFLD (7) and type 2 diabetes, there is concern that the findings are incomplete, and thus potentially misleading, due to the lack of available studies from many countries. These findings highlight the need for additional comprehensive studies across the globe to provide a better characterization of this important health issue.

\section{The importance and challenges of assessing the burden of NAFLD locally and globally}

Prevalence estimates are one of the most important measures of disease burden, and clearly global, regional and local data are important for a variety of reasons. However, it is critical to understand the limitations to the sampling strategy for the patients studied, as well as any variation in the definitions and diagnostic tools used. 
For the current study, which aimed to estimate the prevalence of NAFLD in people with diabetes, large population-based studies, representative of all people with type 2 diabetes, would be the ideal. Yet, these studies, even if the studied sample is representative of the entire population of interest, can only easily incorporate noninvasive measures of the desired outcome (e.g., blood test or imaging). Estimates of NASH and fibrosis, however, are typically derived from patients who had a liver biopsy. These are almost never a representative sample, since they come from patients who are under care, had an indication for liver biopsy and agreed to have one. Hypothetically, these studies could underestimate the true prevalence of $\mathrm{NASH}$ and fibrosis in the population of patients with type 2 diabetes; however, it is more likely they are an overestimate of the severity of disease. In the future, this limitation could be partially addressed by the use of imaging tools [ultrasound- or magnetic resonance (MR)-based] that use measures of liver stiffness as surrogates of liver fibrosis. For estimating the prevalence of NASH, however, the outlook is somewhat discouraging as we currently lack non-invasive tools or biomarkers that can provide accurate measures of this manifestation of the disease.

In addition to the limitations in sampling the population of interest, the variation in diagnostic tools and definitions for NAFLD is worth highlighting. From a histopathological point of view, NAFLD encompasses a combination of interrelated and overlapping manifestations, from fat infiltration, through inflammation and fibrosis, to cirrhosis (8). Clinically, NAFLD is a diagnosis of exclusion which requires assessment and exclusion of excessive alcohol consumption and other liver diseases (e.g., viral hepatitis). The prevalence of these "other conditions", and the extent to which they are excluded, varies across studies. How much this variation affects the prevalence estimates is not easily determined.

Until recently, liver enzymes were used were widely used to screen for liver disease in population-based studies. Although liver enzymes continue to be the most widely available and inexpensive method to identify liver damage in clinical settings and research, studies have shown that normal levels do not adequately exclude the presence of disease (9). Two different imaging methods, ultrasound- or MR-based tools, can now assess fat infiltration and fibrosis. Vibration controlled transient elastography (VCTE) has been FDA approved as a test for the evaluation of liver fat and fibrosis and has a high accuracy for detection of advanced fibrosis (10-12).
Not surprisingly, Younossi et al. demonstrate how widely prevalence estimates of NAFLD vary depending on the diagnostic tool (table S6), from $93 \%$ in liver biopsy studies, to $65 \%$ using blood tests, to $57 \%$ in studies using imaging. Interestingly, MR and ultrasound show similar prevalence estimates of NAFLD (59\% vs. $58 \%$, shown in table 1). As noted above, some of these differences are also due to the sampling frame in the study, particularly for biopsy studies.

\section{Implications of the results}

Despite the limitations of the published studies, there is little doubt that the prevalence of NAFLD is quite high, especially among people with type 2 diabetes. Given the growing evidence that NAFLD will soon be the leading cause of cirrhosis requiring liver transplantation (13), global strategies for prevention, screening and treatment of NAFLD would seem to be needed. We touch on these below.

Prevention strategies for obesity and type 2 diabetes, both key drivers of the NAFLD epidemic, have proven difficult to implement widely. The CDC's National Diabetes Prevention Program has gained traction, and is now a covered program by Medicare, and Medicaid in several states. However, the availability and capacity of programs, and referrals, while growing, remain lower than needed to impact the public health epidemic. However, prevention of diabetes has multiple other benefits and should be a key focus of public health and health system efforts.

Guidelines and recommendations for screening for NAFLD are mixed. On the one hand, the 2017 Diagnosis and Management of NAFLD: Practice Guidance from The American Association for the Study of Liver Diseases (14) does not recommend routine screening for NAFLD in adults (even in those with diabetes or obesity). In contrast, the American Diabetes Association in the Standards of Medical Care in Diabetes 2019 (15), recommends that patients with elevated liver enzymes or fatty liver detected by ultrasound should be evaluated for presence of NASH or liver fibrosis, despite grading the evidence supporting the recommendation as poor $(\mathrm{C})$.

Should we be screening for NAFLD? The WHO outlined the principles of screening five decades ago; these are still widely accepted criteria for the assessment of evidence on benefits, risks and costs of screening. Many of these criteria have now been met for NAFLD, including the importance of the health problem and a recognizable latent or early stage of disease. With the 
advent of VCTE and similar tools, there now exists a screening test with a reasonable level of accuracy-at least for steatosis and fibrosis-that is acceptable and relatively affordable.

The glaring hole in the screening paradigm for NAFLD, is that despite active research, there are still no FDA-approved medications. And while weight loss of significant magnitude has shown efficacy, outside of bariatric surgery, intensive weight loss programs are not covered by insurance in the US. Furthermore, the amount and durability of weight loss from both lifestyle programs and medications are not high, limiting their long-term effectiveness.

Assuming an effective treatment is ultimately identified, there will be a mandate for well-conducted studies to determine whether early detection improves health outcomes, and that the benefits of screening outweigh any potential harms. More evidence will also be needed to understand the natural history of the disease, particularly for those without NASH or significant fibrosis, so that we can identify individuals most likely to benefit from treatment. Finally, work will be required to build the capacity for diagnosis and treatment of a condition that affects millions of patients worldwide.

The results of this study are important, and should serve as an urgent call for studies to fill important gaps in the literature, so that we can begin to address the epidemic of NAFLD across the globe.

\section{Acknowledgments}

Funding: None.

\section{Footnote}

Conflicts of Interest: Both authors have completed the ICMJE uniform disclosure form (available at http://dx.doi. org/10.21037/hbsn.2019.11.28). The authors have no conflicts of interest to declare.

Ethical Statement: The authors are accountable for all aspects of the work in ensuring that questions related to the accuracy or integrity of any part of the work are appropriately investigated and resolved.

Open Access Statement: This is an Open Access article distributed in accordance with the Creative Commons Attribution-NonCommercial-NoDerivs 4.0 International
License (CC BY-NC-ND 4.0), which permits the noncommercial replication and distribution of the article with the strict proviso that no changes or edits are made and the original work is properly cited (including links to both the formal publication through the relevant DOI and the license). See: https://creativecommons.org/licenses/by-nc$\mathrm{nd} / 4.0 \%$.

\section{References}

1. Zelman S. The liver in obesity. AMA Arch Intern Med 1952;90:141-56.

2. Clark JM, Diehl AM. Hepatic steatosis and type 2 diabetes mellitus. Curr Diab Rep 2002;2:210-5.

3. Itoh S, Tsukada Y, Motomura Y, et al. Five patients with nonalcoholic diabetic cirrhosis. Acta Hepatogastroenterol (Stuttg) 1979;26:90-7.

4. Boulet P, Mirouze J, Barjon P, et al. [Non-hemochromatic cirrhosis and diabetes mellitus. (A propos of 25 cases)]. Diabete 1962;10:51-3.

5. Younossi ZM, Koenig AB, Abdelatif D, et al. Global epidemiology of nonalcoholic fatty liver disease-Metaanalytic assessment of prevalence, incidence, and outcomes. Hepatology 2016;64:73-84.

6. Younossi ZM, Golabi P, de Avila L, et al. The global epidemiology of NAFLD and NASH in patients with type 2 diabetes: A systematic review and meta-analysis. J Hepatol 2019;71:793-801.

7. Trepo E, Romeo S, Zucman-Rossi J, et al. PNPLA3 gene in liver diseases. J Hepatol 2016;65:399-412.

8. Brunt EM. Pathology of fatty liver disease. Mod Pathol 2007;20 Suppl 1:S40-8.

9. Mofrad P, Contos MJ, Haque M, et al. Clinical and histologic spectrum of nonalcoholic fatty liver disease associated with normal ALT values. Hepatology (Baltimore, Md) 2003;37:1286-92 .

10. Tang A, Cloutier G, Szeverenyi NM, et al. Ultrasound Elastography and MR Elastography for Assessing Liver Fibrosis: Part 1, Principles and Techniques. AJR Am J Roentgenol 2015;205:22-32.

11. Tang A, Cloutier G, Szeverenyi NM, et al. Ultrasound Elastography and MR Elastography for Assessing Liver Fibrosis: Part 2, Diagnostic Performance, Confounders, and Future Directions. AJR Am J Roentgenol 2015;205:33-40.

12. Barr RG, Ferraioli G, Palmeri ML, et al. Elastography Assessment of Liver Fibrosis: Society of Radiologists in Ultrasound Consensus Conference Statement. Radiology 
2015;276:845-61.

13. Wong RJ, Aguilar M, Cheung R, et al. Nonalcoholic steatohepatitis is the second leading etiology of liver disease among adults awaiting liver transplantation in the United States. Gastroenterology 2015;148:547-55.

14. Chalasani N, Younossi Z, Lavine JE, et al. The diagnosis and management of nonalcoholic fatty liver disease: Practice guidance from the American Association for the Study of Liver Diseases. Hepatology.2018;67:328-57.

15. American Diabetes A. 4. Comprehensive Medical Evaluation and Assessment of Comorbidities: Standards of Medical Care in Diabetes-2019. Diabetes Care 2019;42:S34-45.

Cite this article as: Lazo M, Clark JM. Nonalcoholic fatty liver disease and type 2 diabetes: a burgeoning problem with unclear solutions. HepatoBiliary Surg Nutr 2020;9(4):514-517. doi: $10.21037 /$ hbsn.2019.11.28 